\title{
Mortalidade neonatal no Brasil no período de 2004 a 2014
}

\author{
Neonatal mortality in Brazil in the period from 2004 to 2014 \\ Mortalidad neonatal en Brasil en el período de 2004 a 2014
}

Suanne Macêdo de Mendonça ${ }^{1,}$ Ridalva Dias Martins Felzemburgh ${ }^{1}$, Josely Bruce dos Santos ${ }^{2 *}$.

\begin{abstract}
RESUMO
Objetivo: Estimar as taxas de mortalidade neonatal no Brasil entre os anos de 2004 a 2014 e sua variação ao longo dos anos. Estimar as taxas de mortalidade neonatal por regiões brasileiras e sua variação entre as regiões brasileiras ao longo do período citado. Métodos: Trata-se de um Estudo ecológico realizado com dados secundários extraídos no departamento de informática do Sistema Único de Saúde (DATASUS), no Sistema de Informação de Mortalidade (SIM) e no Sistema de Informação de Nascidos Vivos (SINASC). O período do estudo se deu de 2004 a 2014, as variáveis estudadas foram o número de nascidos vivos e o número de óbitos de crianças de 0 a 27 dias. Resultados: As taxas de mortalidade neonatal variaram ao longo dos anos entre o máximo de 11,86 no ano de 2004 e o mínimo de 8,97 no ano de 2014 . Isso reflete uma tendência decrescente para o Brasil ao longo dos anos. A região Sul tem uma mínima obtida de 7,56 no ano de 2014. A região Nordeste variou de 13,80 em 2004 a 10,31 no ano de 2014. Conclusão: A partir do quadro epidemiológico brasileiro, a diminuição da mortalidade neonatal ainda é um desafio para os serviços de saúde, a sociedade e o governo. Para reduzir essas taxas é fundamental a intervenção nos diversos fatores que afetam a mortalidade neonatal.
\end{abstract}

Palavras-chave: Mortalidade infantil, Registro de mortalidade, Sistemas de informação.

\begin{abstract}
Objective: To estimate neonatal mortality rates in Brazil between 2004 and 2014 and their variation over the years; To estimate the neonatal mortality rates by Brazilian regions and their variation among the Brazilian regions during the mentioned period. Methods: An ecological study based on secondary data obtained from the computer department of the Brazilian National Health System (DATASUS). Data collection was done through consultations in the Mortality Information System (SIM) and in the Live Birth Information System (SINASC). The study period was from 2004 to 2014, the variables studied were the number of live births and the number of deaths of children from 0 to 27 days. Results: Neonatal mortality rates varied over the years from a high of 11.86 in 2004 to a low of 8.97 in 2014. This reflects a downward trend for Brazil over the years. The South region has a minimum obtained of 7.56 in the year 2014. The Northeast region varied from 13.80 in 2004 to 10.31 in the year 2014. Conclusion: From the Brazilian epidemiological framework, the reduction of neonatal mortality still is a challenge for health services, society and government. In order to reduce these rates intervention on the various factors that affect neonatal mortality is fundamental.
\end{abstract}

Key words: Infant Mortality, Mortality Records, Information systems.

1 Universidade Federal da Bahia, Salvador, Bahia.

2Universidade Federal da Bahia e Centro Universitário Jorge Amado, Salvador, Bahia.

*E-mail: joselybruce3@gmail.com

SUBMETIDO EM: 9/2018

ACEITO EM: 10/2018

PUBLICADO EM: 12/2018

REAS/EJCH | Vol. 11 (2) | e142 | DOI: https://doi.org/10.25248/reas.e142.2019 Página 1 de 7 


\section{RESUMEN}

Objetivo: Estimar las tasas de mortalidad neonatal en Brasil entre los años 2004 a 2014 y su variación a lo largo de los años; Estimar las tasas de mortalidad neonatal por regiones brasileñas y su variación entre las regiones brasileñas a lo largo del período citado. Métodos: Estudio ecológico realizado a partir de datos secundarios obtenidos en el departamento de informática del Sistema Único de Salud (DATASUS). La recolección de datos fue hecha a partir de consultas en el Sistema de Información de Mortalidad (SIM) y en el Sistema de Información de Nacidos Vivos (SINASC). El período del estudio se dio de 2004 a 2014, las variables estudiadas fueron el número de nacidos vivos y el número de muertes de niños de 0 a 27 días. Resultados: Las tasas de mortalidad neonatal variaron a lo largo de los años entre el máximo de 11,86 en el año 2004 y el mínimo de 8,97 en el año 2014. Esto refleja una tendencia decreciente hacia Brasil a lo largo de los años. La región Sur tiene una mínima obtenida de 7,56 en el año 2014. La región Nordeste varió de 13,80 en 2004 a 10,31 en el año 2014. Conclusión: A partir del cuadro epidemiológico brasileño, la disminución de la mortalidad neonatal aún es un desafío para los servicios de salud, la sociedad y el gobierno. Para reducir estas tasas es fundamental la intervención en los diversos factores que afectan la mortalidad neonatal.

Palabras clave: Mortalidad Infantil, Registros de Mortalidad, Sistemas de información.

\section{INTRODUÇÃO}

A taxa de Mortalidade Neonatal (TMN) é um importante indicador, pois é o principal componente da mortalidade infantil, refletindo transformações sociais de regiões, condições de vida da população e da atenção à saúde no país, e a partir dos resultados pode haver um controle e redução dos seus níveis através de ações pertinentes (LANSKY et al., 2014).

Em um estudo de coorte realizado em 2004 no município de pelotas (MS), foram registrados 94 óbitos, 82 antes do primeiro ano de vida completo, o que se refere ao coeficiente de mortalidade infantil de 19,4 óbitos por 1.000 NV (GORGOT et al.,2011). A redução da mortalidade infantil configura-se como prioritário e estratégico para a redução da mortalidade na infância para o país (LIMA et al.,2013).

De acordo com Lima et al. (2013), no Brasil, em 2009 foram notificados em torno de 40.000 óbitos de crianças menores de um ano. Estima-se que $62 \%$ destes óbitos são considerados evitáveis.

Para Buss e Pelegrini Filho (2007) no Brasil, a mortalidade infantil tem uma relação direta com a condição financeira das famílias, a escolaridade, as condições de moradia e a situação social da família, mostrando-se em números que indicam essa relação, tendo visto que a mortalidade infantil é de 16 por mil nascidos vivos entre os ricos contra 35 por mil nascidos vivos entre os pobres.

A mortalidade neonatal no Brasil ainda se encontra com altas taxas desde a década de 1990, taxas essas que são reflexo de diversos fatores, tais como biológicos, assistenciais e socioeconômicos que refletem as condições de vida da população Brasileira. É de extrema importância que atitudes preventivas sejam tomadas em prol de uma melhor condição de vida da população e dos serviços de saúde, para que sejam evitadas as mortes desses indivíduos (LANSKY et al., 2014).

Trata-se de um problema atual, que gera muitos impactos para a saúde pública e que poderiam ser modificados. Nesse contexto, tal estudo justifica-se por contribuir para estimativa das taxas de mortalidade neonatal no Brasil entre os anos de 2004 a 2014 e identificação de como essas taxas variam entre as regiões brasileiras no período citado. As questões norteadoras do estudo foram: Quais as taxas de mortalidade neonatal no Brasil entre os anos de 2004 a 2014? E como essas taxas variaram entre as regiões brasileiras no período citado?

Desta forma, o presente estudo tem como objetivo estimar as taxas de mortalidade neonatal no Brasil entre os anos de 2004 a 2014, além de identificar como essas taxas variaram ao longo dos anos, estimar as taxas de mortalidade neonatal por regiões brasileiras e identificar como essas taxas variaram entre as regiões brasileiras ao longo do período citado. 


\section{MÉTODO}

Trata-se de um estudo ecológico realizado com dados secundários extraídos no departamento de informática do Sistema Único de Saúde (DATASUS), no Sistema de Informação de Mortalidade (SIM) e no Sistema de Informação de Nascidos Vivos (SINASC). O período do estudo ocorreu entre os anos de 2004 a 2014 , a população do estudo foram crianças entre 0 a 27 dias de vida. As variáveis estudadas foram o número de nascidos vivos e o número de óbitos de crianças de 0 a 27 dias de vida.

Para obtenção dos resultados foi realizado o cálculo da taxa de mortalidade por regiões brasileiras para o período estudado. Os dados foram apresentados em duas tabelas e um gráfico. Por se tratar de fonte secundária e de domínio público, não foi necessária submissão ao comitê de ética em pesquisa.

\section{RESULTADOS E DISCUSSÃO}

As taxas de mortalidade neonatal variaram ao longo dos anos entre o máximo de 11,86 no ano de 2004 e o mínimo de 8,97 no ano de 2014. De acordo com a tabela 1, isso reflete uma tendência decrescente para o Brasil ao longo dos anos. A mortalidade infantil no Brasil encontra-se em queda contínua desde 1990. Houve uma redução significativa na taxa de mortalidade infantil, que passou de 47,1 óbitos a cada mil bebês nascidos vivos para 19,3 em 2007, o que representa uma redução de $59,7 \%$ dessa taxa (BRASIL, 2011).

Apesar do Brasil manter as taxas de mortalidade infantil em declínio, conforme observa-se os dados de Garcia e Santana (2011), que em 1980 a taxa de mortalidade infantil era de 78,5 óbitos por mil nascidos vivos enquanto que em 2008 era em torno de 19,1 óbitos por mil nascidos vivos, manteve-se superior em relação ao Japão, Portugal, Suécia dentre outros, cuja média é de 3 óbitos por mil nascidos vivos. Alguns outros países da América Latina também apresentavam essas taxas inferiores as do Brasil, a exemplo da Argentina, Chile e Costa Rica que possuíam taxas de 11,07, 10 por mil nascidos vivos respectivamente.

Em 2000 houve uma preocupação mundial a mais sobre a mortalidade neonatal, ganhando visibilidade através de um encontro com os grandes líderes mundiais para estabelecerem o compromisso com algumas metas importantes para a população mundial, tendo em vista as melhores condições de vida. Diante disso foi feito um plano de ação (Objetivos de Desenvolvimento do Milênio) sobre metas que os países teriam que chegar para melhorar a qualidade de vida no geral de sua população. Em 2015 os líderes se reuniram para reavaliar essas metas, já que o prazo para atingir as metas foi encerrado. A partir daí foi criada uma agenda até 2030 com novas metas a serem alcançadas, dentre elas estão a extinção das mortes evitáveis de recém-nascidos e menores de cinco anos, com o objetivo maior de reduzir a mortalidade neonatal para taxas de até 12 por 1000 nascidos vivos (ONU, 2015).

Relacionando a tabela 1 ao objetivo de redução da mortalidade neonatal para taxas de 12 por 1000 nascidos vivos, considera-se que o Brasil já tenha alcançado essa meta, pois desde 2004 o Brasil já possui uma taxa de mortalidade neonatal inferior aos valores exigidos atualmente, porém sabe-se que o Brasil é um país que tem grandes desigualdades regionais que refletem a qualidade de vida da população e que precisam ser ao menos diminuídas. Segundo França e Lansky (2009) apesar da evidente diminuição, as Taxas de Mortalidade Infantil (TMI) ainda são elevadas no Brasil, apresentando diferenças na distribuição entre as regiões brasileiras. Percebe-se uma maior concentração de óbitos nas regiões Nordeste e Amazônia Legal onde, ao longo do período de 2000 a 2007, ocorreu metade do total de óbitos infantis do país. Essas desigualdades também acontecem em um mesmo estado, pois a TMI tem uma tendência a aumentar conforme se afasta da capital para a área metropolitana e o interior.

De acordo com Oliveira et al. (2013) há um coeficiente de mortalidade neonatal alto no país confirmando os resultados de uma pesquisa anterior, realizada em 2007, onde pôde ser observada uma taxa de 10,7 óbitos por mil nascidos vivos, taxa essa que se mantém 2,5 vezes maior que a média dos Estados Unidos 4 óbitos por 1.000 nascidos vivos) em 2006 e em torno de 10 vezes maior que a do Japão (1 óbito por 1.000 nascidos vivos), em 2004. 
Uma forma de esclarecer mais sobre as taxas de mortalidade neonatal seria identificar por componente, visto que desse modo poderia saber mais detalhadamente sobre o real motivo de algumas variáveis se manterem altas e outras baixas e realizar mais relações entre as variáveis e os componentes. Dessa forma conseguindo, a partir desses dados, implementar alguma política pública voltada para a melhoria dos índices que fazem com que essas taxas fiquem no geral abaixo do esperado.

De acordo com França e Lansky (2009) os maiores declínios da Taxa de mortalidade infantil se deram na mortalidade pós-neonatal com $8,1 \%$, enquanto que a mortalidade neonatal precoce foi apenas de $2,9 \%$. Logo, pode-se inferir que esses óbitos podem ter uma relação com a falta de uma assistência ao pré-natal, parto e puerpério adequada, porém deve-se fazer mais estudos para conseguir perceber todos os fatores que levam a essa situação de pouco declínio da mortalidade neonatal.

A região Sul tem uma mínima obtida de 7,56 no ano de 2014, conforme a tabela 2. Percebe-se que, juntamente com as outras regiões, há uma tendência decrescente e que a região Sul tem uma peculiaridade de ser a região com as menores taxas de mortalidade neonatal, se mantendo sempre abaixo quando se compara a nível de Brasil.

As regiões Sul e Sudeste apresentam melhores resultados com relação as taxas de mortalidade neonatal, dentre eles, os estados que apresentaram menores taxas foram os que possuíam um quantitativo menor de mães com menos de oito anos de escolaridade. A educação está relacionada a inserção da população no mercado de trabalho, pois é o meio de preparar as pessoas para ocupar as vagas de emprego e assim atingirem melhores salários. Dessa forma o sistema de educação brasileiro reforça a estrutura social existente, apesar dos avanços ocorridos no acesso ao ensino fundamental e médio, as diferenças regionais ainda são muito grandes além de terem uma maior taxa de evasão escolar nos estados do Norte e Nordeste com relação os Sul e Sudeste (BRASIL, 2010).

A partir da tabela 2, percebe-se que as taxas de mortalidade neonatal variam de uma máxima obtida de 13,80 no ano de 2004 para a região Nordeste. A região Nordeste variou de 13,80 em 2004 a 10,31 no ano de 2014. Apresenta-se com uma tendência decrescente ao longo dos anos, apesar disso quando se faz relação com as taxas a nível de Brasil fica claro que as taxas do Nordeste se mantêm níveis acima das taxas do país, a partir desses dados fica claro que o Brasil é um país com altos níveis de desigualdades regionais.

Sabe-se que as desigualdades regionais no país, principalmente no meio Norte-Nordeste, regiões que possuem os piores indicadores de saúde e socioeconômicos, decorrem provavelmente do processo de ocupação e expansão brasileiro. A ocupação que inicialmente foi realizada em áreas litorâneas e posteriormente foi utilizando de áreas do interior para produção agrícola, gerou uma concentração dos investimentos e atividades no eixo centro-sul do país. Ao longo dos anos foi ocorrendo mudanças e incentivos através de políticas que visavam o desenvolvimento das áreas mais pobres, isso fez com que houvesse um povoamento maior nas áreas do interior do país. Essas políticas melhoraram a distribuição regional do PIB per capita, porém não foi suficiente para descentralizar a distribuição da região Centro-sul, estabelecendo dessa forma os menores níveis de rendimento para o semiárido nordestino e o estado do Amazonas (BRASIL, 2005).

As regiões Norte e Nordeste possuem um maior número de subnotificação de óbitos gerais e particularmente infantis com dimensões maiores, podendo causar subestimação das taxas, pois deixa de contabilizar inúmeros casos de grupos sociais mais necessitados da sociedade. Por serem regiões com piores níveis de desenvolvimento humano e tecnológico e sabendo que esses fatores são determinantes para a qualidade dos registros e do melhor preenchimento dos documentos, fica claro que falta uma capacitação para que esses profissionais tenham melhores condições tanto técnicas quanto de perceberem a importância de ter a completude nos dados para que dessa forma sejam implementadas políticas adequadas (IBGE, 2009). 
Tabela 1: Taxas de Mortalidade Neonatal por Ano para o Brasil.

\begin{tabular}{lccccccccccc}
\hline \multicolumn{1}{c}{ Variável } & $\mathbf{2 0 0 4}$ & $\mathbf{2 0 0 5}$ & $\mathbf{2 0 0 6}$ & $\mathbf{2 0 0 7}$ & $\mathbf{2 0 0 8}$ & $\mathbf{2 0 0 9}$ & $\mathbf{2 0 1 0}$ & $\mathbf{2 0 1 1}$ & $\mathbf{2 0 1 2}$ & $\mathbf{2 0 1 3}$ & $\mathbf{2 0 1 4}$ \\
\hline Nascidos vivos & 3026548 & 3035094 & 2944928 & 2891328 & 2934828 & 2934828 & 2861868 & 2913160 & 2905789 & 2904027 & 2979259 \\
Total de óbitos & 35890 & 34382 & 32597 & 30821 & 30179 & 29212 & 27687 & 27534 & 27113 & 26730 & 26746 \\
Taxa de Mortalidade Neonatal $^{*}$ & 11,86 & 11,33 & 11,07 & 10,66 & 10,28 & 10,14 & 9,67 & 9,45 & 9,33 & 9,20 & 8,97 \\
\hline
\end{tabular}

${ }^{*}$ Por 1.000 nascidos vivos

Fonte: Dados da pesquisa, 2018.

Tabela 2: Taxas de Mortalidade* Neonatal por Ano por Regiões do Brasil.

\begin{tabular}{|c|c|c|c|c|c|c|c|c|c|c|c|}
\hline Região & 2004 & 2005 & 2006 & 2007 & 2008 & 2009 & 2010 & 2011 & 2012 & 2013 & 2014 \\
\hline Norte & 13,09 & 12,30 & 12,74 & 12,03 & 11,53 & 11,91 & 11,46 & 10,88 & 10,69 & 10,62 & 10,32 \\
\hline Nordeste & 13,80 & 13,32 & 12,91 & 12,68 & 11,81 & 11,74 & 11,16 & 10,79 & 10,78 & 10,94 & 10,31 \\
\hline Sudeste & 10,71 & 10,20 & 9,91 & 9,44 & 9,31 & 9,12 & 8,66 & 8,51 & 8,39 & 8,19 & 8,14 \\
\hline Sul & 9,98 & 9,41 & 9,16 & 8,73 & 8,79 & 8,14 & 7,95 & 8,08 & 7,72 & 7,25 & 7,56 \\
\hline Centro-Oeste & 11,65 & 10,99 & 10,51 & 10,07 & 9,79 & 9,84 & 9,59 & 9,51 & 9,63 & 9,39 & 8,97 \\
\hline
\end{tabular}

*Por 1.000 nascidos vivos

Fonte: Dados da pesquisa, 2018. 
Muitos estudos citam essa baixa confiabilidade dos Sistemas de Informação em Saúde e trazem como causa o alto grau de omissão dos campos dos documentos que os alimentam, a inconsistência de dados. Ressalta-se que a qualidade dos registros depende das condições de desenvolvimento humano e tecnológico de cada região (MASCARENHAS e GOMES, 2011).

No estudo realizado por Silva et al. (2014), foi observado que o sistema de informações sobre mortalidade apresentava lacunas em diversas variáveis tais como: raça/cor, idade da mãe, escolaridade da mãe, entre outros, sendo que a variável número da Declaração de Nascimento teve a pior completude de informações.

De acordo com o Gráfico 1, fica perceptível a tendência decrescente das taxas de mortalidade do Brasil. Sendo que as regiões sul e sudeste se mantêm abaixo dos níveis do Brasil ao longo de todo o período estudado. Diferente das outras regiões, as regiões Norte e Nordeste se mantêm acima da taxa de mortalidade neonatal do Brasil durante todos os anos. Na região Centro-Oeste percebe-se um declínio ao longo dos anos, se mantendo até o ano de 2010 abaixo do nível das taxas do Brasil, porém a partir de 2011 essas taxas ficam mais elevadas do que o Brasil.

Gráfico 1: Taxas de Mortalidade Neonatal por Ano para o Brasil e Regiões.

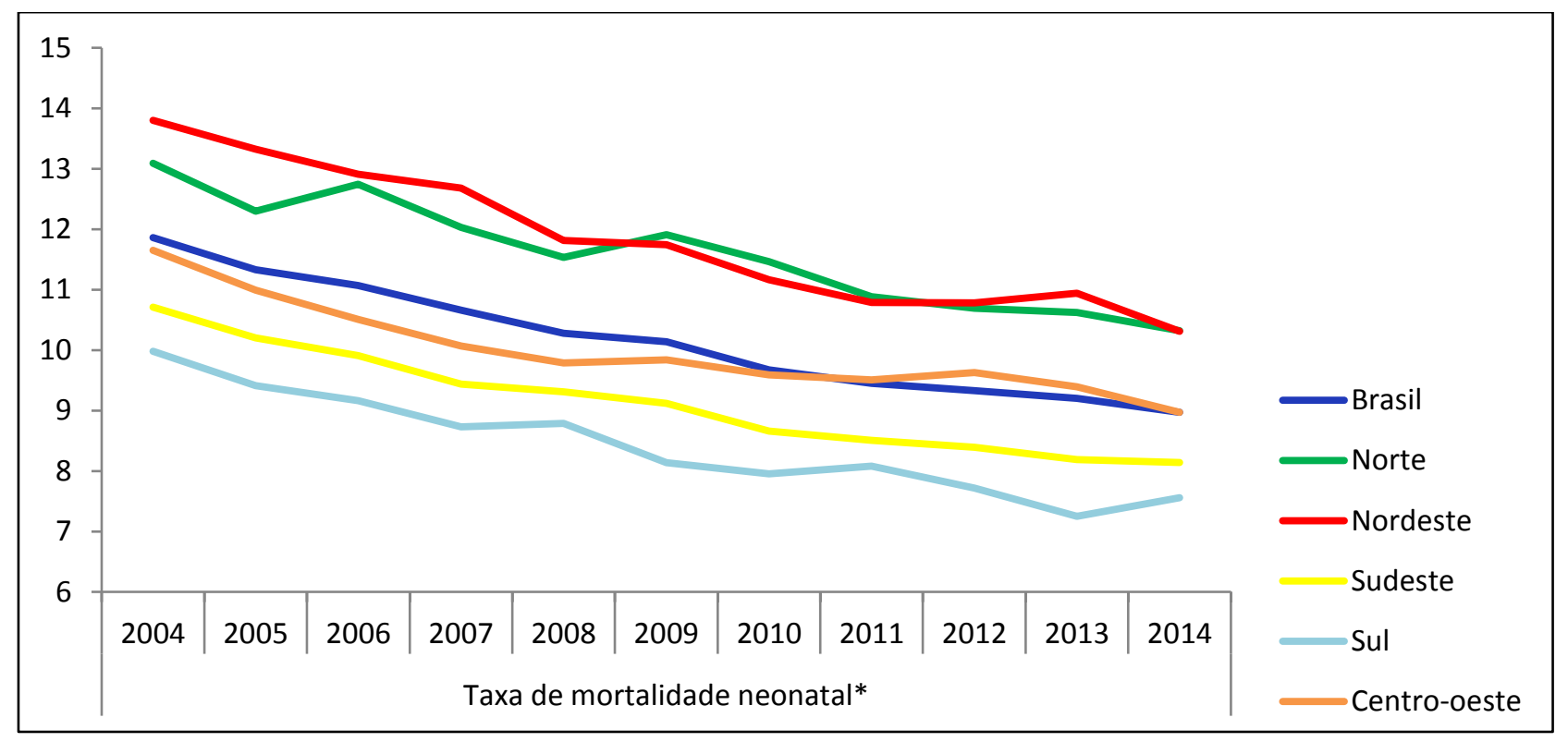

Fonte: dados da pesquisa, 2018.

\section{CONCLUSÂO}

A partir dos resultados obtidos nessa pesquisa, juntamente com os artigos estudados, visando o quadro epidemiológico brasileiro, sabe-se que a diminuição da mortalidade neonatal ainda é um grande desafio para o governo, serviços de saúde e sociedade, pois se apresenta como um reflexo tanto como de iniquidades sociais quanto de acesso igualitário a serviços de saúde de qualidade.

As diferenças no acesso e na distribuição da saúde no País sinalizam uma necessidade de políticas que visem à diminuição das desigualdades, melhorando o acesso da população aos serviços de saúde, aumentando dessa forma as taxas de utilização de tais serviços, principalmente nas regiões Norte e Nordeste, onde foram encontradas as maiores iniquidades, garantindo dessa forma a equidade na distribuição entre a população. 
Para que seja alcançada a redução das taxas de mortalidade neonatal é de fundamental importância que se faça uma intervenção nos diversos fatores que estão ligados a mortalidade neonatal, tais como: fatores econômicos, sociais e assistenciais, desde a atenção básica à alta complexidade, atendendo integralmente ao público materno-infantil.

Chama-se a atenção sobre a importância da sensibilização dos profissionais de saúde com relação à subnotificação que podem ser controlados por atestados de óbitos e declarações de nascimentos. Deve-se haver uma modificação no sistema para que sejam exigidos dados mais completos e dessa forma haja dados mais fidedignos.

\section{REFERÊNCIAS}

1. BRASIL. Estatísticas vitais - mortalidade e nascidos vivos. Brasília: Ministério da Saúde. Datasus; 2011. Disponível em: http://www.datasus.gov.br/indicadoresdesaude/estatisticasvitais. Acesso em 21 Set. 2016

2. BRASIL. Pacto pela Redução da Mortalidade Infantil Nordeste-Amazônia Legal 2009-2010. 3v. Brasília: Ministério da Saúde, 2010.

3. BRASIL. Política Nacional de Desenvolvimento Regional. Brasília: Ministério da Integração Nacional. Secretaria de Políticas de Desenvolvimento Regional, 2005. Disponível em: http://www.unc.br/mestrado/mestrado_materiais/10.03.08__PNDR_texto_prova_seletiva.pdf. Acesso em 13 Ago. 2016.

4. BUSS PM, PELLEGRINI Filho A. A saúde e seus determinantes sociais. Physis, 2007; 17(1):77-93.

5. FRANÇA E, LANSKY S. Mortalidade infantil neonatal no Brasil: Situação, tendências e perspectivas. In: Rede Interagencial de Informações para Saúde (org). Demografia e saúde: contribuição para análise de situação e tendências. Brasília: Organização PanAmericana da Saúde, 2009; 83-112.

6. GARCIA LP, SANTANA LR. Evolução das desigualdades socioeconômicas na mortalidade infantil no Brasil, 1993-2008. Ciênc. Saúde coletiva, 2011; 16(9): 3717-3728.

7. GORGOT LRMR, SANTOS I, VALE $N$ et al. Óbitos evitáveis até 28 meses de idade entre crianças da coorte de nascimentos de Pelotas de 2004. Ver Saúde Pública, 2011;45(2):334-42.

8. INSTITUTO BRASILEIRO DE GEOGRAFIA E ESTATíSTICA (IBGE). Indicadores Sociodemográficos e de Saúde no Brasil, 2009. Disponível em: http://www.ibge.gov.br/home/estatistica/populacao/indic sociosaude/2009/default.shtm. Acesso em 03 de Set. 2016

9. LANSKY S, FRICHE AAL, SILVA AAM et al. Pesquisa Nascer no Brasil: perfil da mortalidade neonatal e avaliação da assistência à gestante e ao recém-nascido. Cadernos de Saúde Pública, 2014; 30(Sup): S192-S207.

10. LIMA MCBM, OLIVEIRA GS, LYRA CO et al. A desigualdade espacial do Baixo Peso ao Nascer no Brasil. Ciência \& Saúde Coletiva, 2013; 18(8):2443-2452.

11. MASCARENHAS MDM, GOMES KRO. Confiabilidade dos dados do Sistema de Informações sobre Nascidos Vivos em Teresina, Estado do Piauí, Brasil - 2002. Ciência \& Saúde Coletiva, 2011; 16(supl. 1): 1233-1239.

12. OLIVEIRA GS, Lima MCBM, Lyra CO et al. Desigualdade espacial da mortalidade neonatal no Brasil: 2006 a 2010 . Ciência \& Saúde Coletiva, 2013; 18(8):2431-2441.

13. ORGANIZAÇÃO DAS NAÇÕES UNIDAS (ONU). Transformando Nosso Mundo: A Agenda 2030 para o Desenvolvimento Sustentável. 2015. Disponível em: https://nacoesunidas.org/pos2015/agenda2030/. Acesso em 29 de Set de 2016.

14. SILVA LP, MOREIRA CMM, AMORIM MHC et al. Avaliação da qualidade dos dados do Sistema de Informações sobre Nascidos Vivos e do Sistema de Informações sobre Mortalidade no período neonatal, Espírito Santo, Brasil, de 2007 a 2009. Ciência \& Saúde Coletiva, 2014; 19(7):2011-2020. 